\title{
OH-Initiated Oxidation of $\boldsymbol{m}$-Xylene on Black Carbon Aging
}

\author{
Song Guo*1,2, Min Hu ${ }^{1}$, Yun Lin $^{2}$, Mario Gomez-Hernandez ${ }^{3}$, Misti L. Zamora ${ }^{2}$, \\ Jianfei Peng ${ }^{2}$, Donald R. Collins ${ }^{2}$, Renyi Zhang ${ }^{* 1,2,3}$ \\ ${ }^{1}$ State Key Joint Laboratory of Environmental Simulation and Pollution Control, College of \\ Environmental Sciences and Engineering, Peking University, Beijing, 100871, China \\ ${ }^{2}$ Department of Atmospheric Sciences, Texas A\&M University, College Station, TX 77843, USA \\ ${ }^{3}$ Department of Chemistry, Texas A\&M University, College Station, TX 77843, USA
}

Corresponding Authors:

Song Guo: guosong@pku.edu.cn; Renyi Zhang: renyi-zhang@tamu.edu

\section{Supporting Information:}

This supporting information includes 4 figures. 

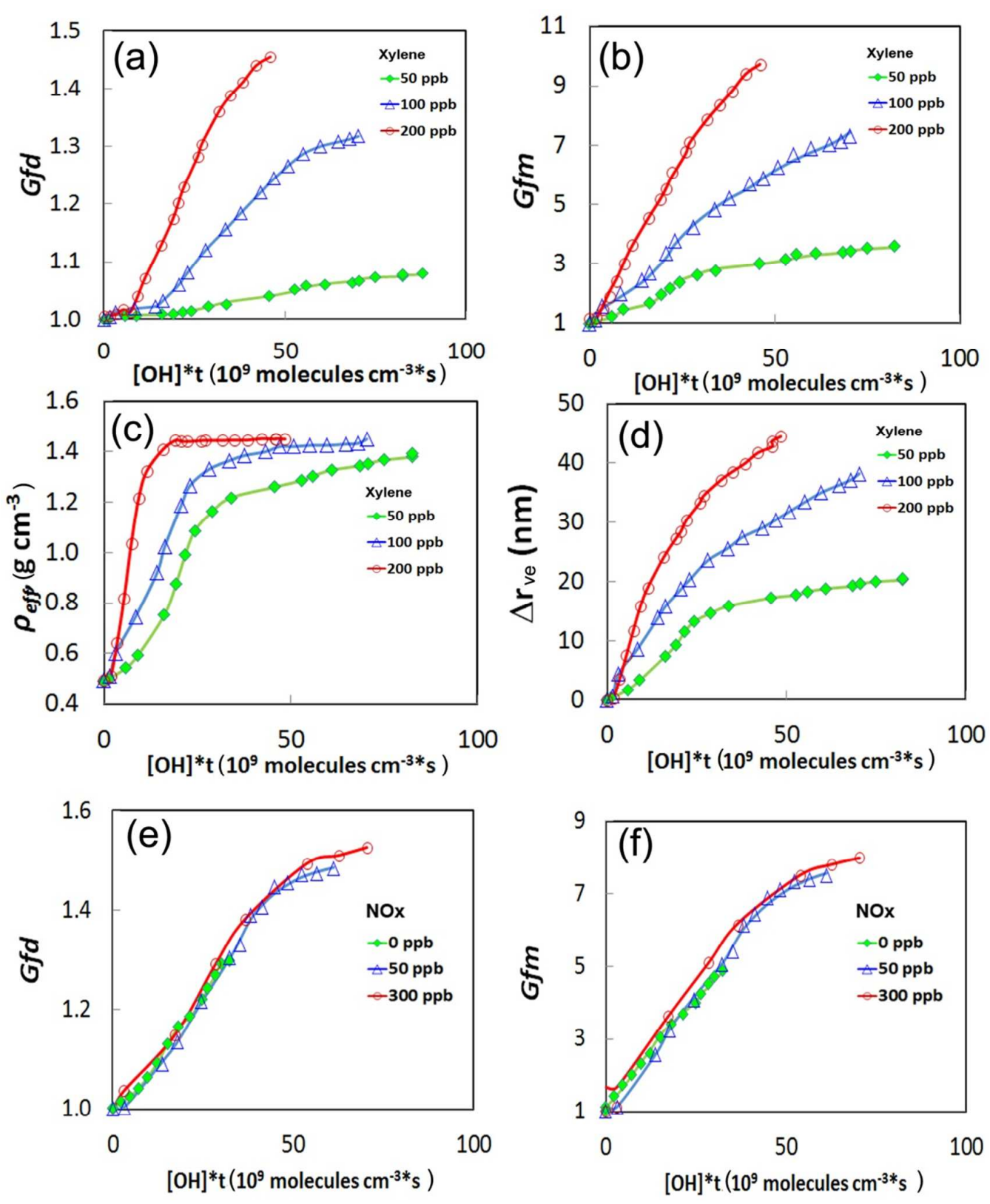

Figure S1. Changes in $\mathrm{BC}$ particle properties with the $\mathrm{OH}$ exposure time under different initial $m$-xylene and $\mathrm{NO}_{\mathrm{x}}$ concentrations. 

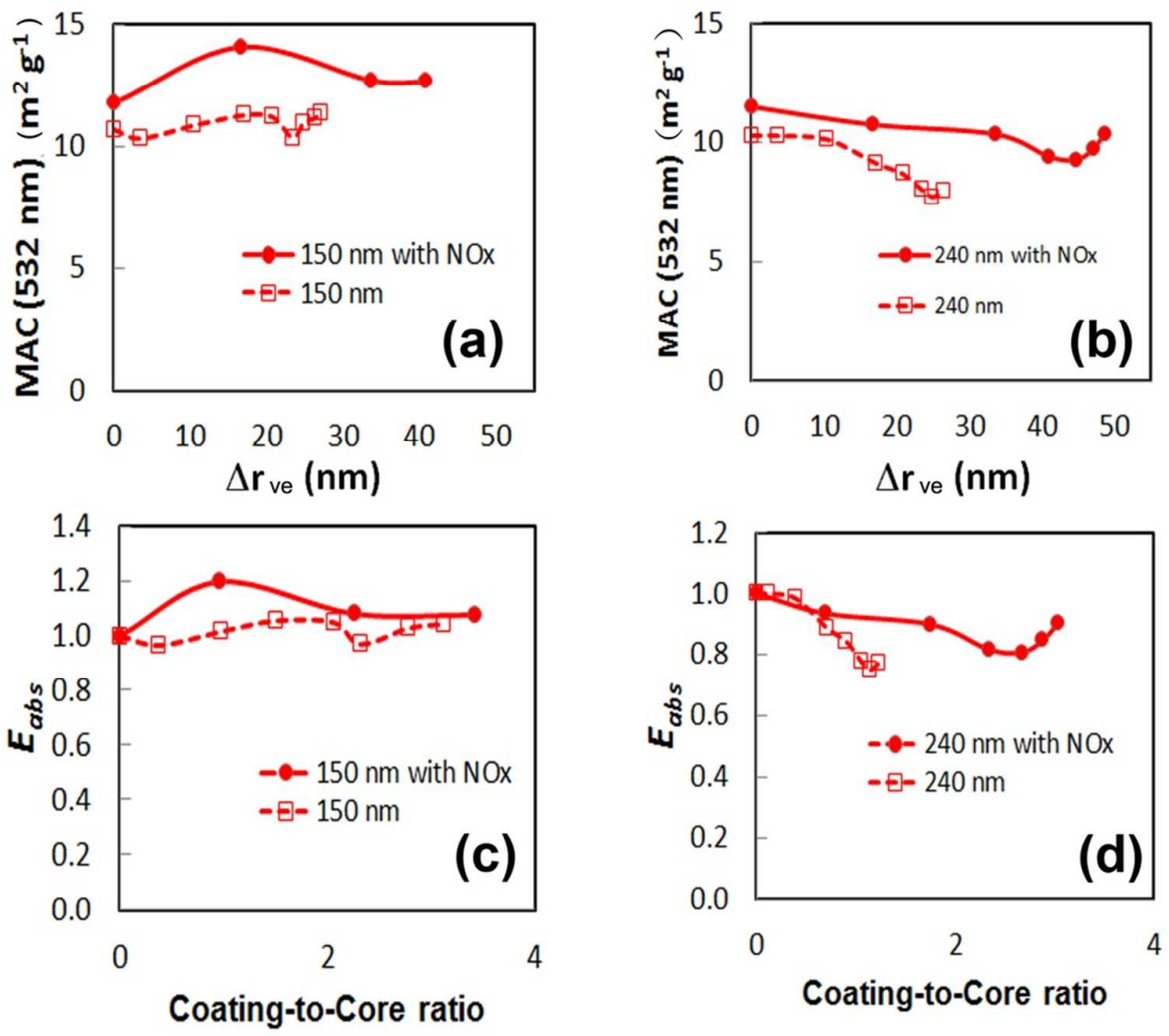

Figure S2. Variation of the mass absorption cross-section (MAC) with the coating thickness $\left(\Delta \mathrm{r}_{\mathrm{ve}}\right)$ and the MAC enhancement with the coating to core mass ratio. 

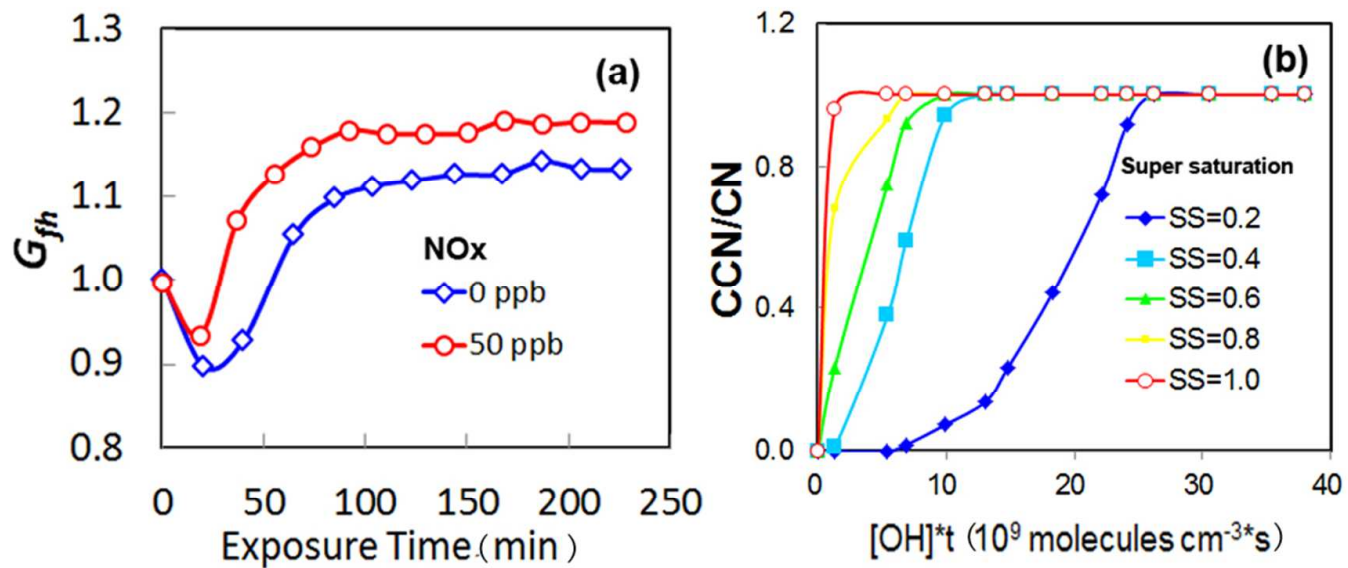

Figure S3. Variation of the hygroscopic growth factor with the reaction time (a), and the variation of the $\mathrm{CCN}$ fraction with the integrated $\mathrm{OH}$ exposure time (b). 

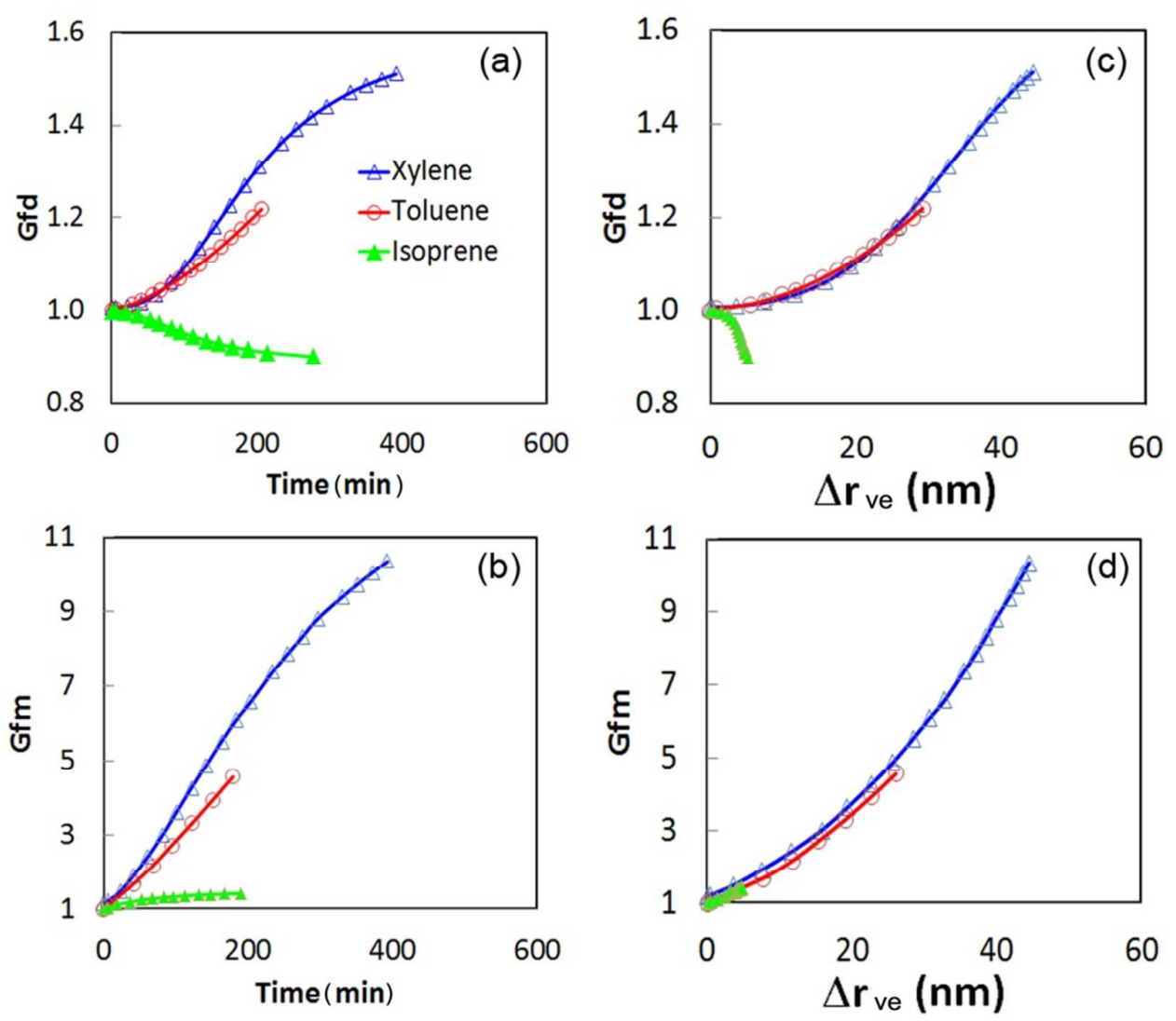

Figure S4. Comparison in the evolution of BC particle properties coated by the oxidation products from the $m$-xylene- $\mathrm{OH}$ with those from previous studies for toluene-OH (ref 27) and isoprene-OH (ref 28) reactions. The experimental conditions are: $D_{p}=100 \mathrm{~nm},[m$-xylene $]=200 \mathrm{ppb}$, [toluene] $=200 \mathrm{ppb}$, and [isoprene $]=340$ ppb. 